\title{
Editorial
}

Pathobiology

\section{Clinical Relevance of Intra-Tumour Heterogeneity}

\author{
Bettina Borisch $^{a}$ Giorgio Stanta ${ }^{b}$ \\ ${ }^{a}$ BioCampus, Institute of Global Health, University of Geneva, Geneva, Switzerland; \\ ${ }^{b}$ Department of Medical Sciences, University of Trieste, Trieste, Italy
}

Intra-tumour heterogeneity (ITH), i.e., that within the same tumour, cells can show different morphological and phenotypic profiles, including cellular morphology, gene expression, metabolism, motility, proliferation, and metastatic potential, has been known but has not attracted very much attention in practical cancer diagnostics for a long time. Years ago, we showed that tracing clonal ITH would enable the study of the hierarchy of mutational events in cancers where no premalignant lesions can be harvested. Most importantly, our previous study [1] indicated that clonal ITH might lead to sampling errors in the molecular diagnosis of cancer biopsy specimens when using mini- or microsatellite markers. Due to the simple "clonal" nature of their tumour cells, neoplastic lesions of the haematopoietic system have been the first to be investigated thoroughly [2]. Since then, the methods of investigation have evolved and allow the topic to be addressed in a more practical way. Understanding and characterizing heterogeneity can lead to a better knowledge of the causes and progression of disease. Given the fact that under therapy heterogeneic tumours may exhibit different sensitivities to cytotoxic drugs among different clonal populations, tumour heterogeneity needs to be included in the diagnostic process. Even without applying specific

\section{KARGER}

๑) 2018 S. Karger AG, Basel

E-Mail karger@karger.com

www.karger.com/pat biomarkers for heterogeneity, the simple level of heterogeneity can itself be used as a biomarker since more heterogeneous tumours may be more likely to contain treatment-resistant subclones. Today, experimental approaches to identify and characterize tumour heterogeneity exist. It is challenging to get the translation into diagnosis and adapted treatment done. This special issue of Pathobiology is devoted to this part.

This Pathobiology issue features a series of papers about ITH in different types of cancer. The chairs of the different tumour working groups of the European Society of Pathology (ESP), or someone on their behalf, were invited to give their reviews and their opinions on the specific types of ITH, even if their papers do not represent the official position of the ESP or working group. The papers have been written from a practical point of view for the clinical application of cancer diagnostics in the present or near future. Indeed, we have to consider that the complaints about irreproducibility in clinical research are also connected with the fact that ITH is very often not taken into consideration. It is therefore important to try to standardize the methods to be applied as a first and starting point for a common standardization of ITH methods - and therefore comparability of results - in the 
future. Regarding ITH, the current literature reveals that common patterns can be found in different cancer types, but important differences are also reported. The knowledge in the field is still a highly dynamic process. This is related to the continuous developments of present-day pathology and oncology and to the necessity to apply the new knowledge to diagnostics and clinical oncology as soon as possible.

This collection of ITH reviews and considerations paves the way for wider applications and standardization of ITH topics in the future. The papers include different approaches and different points of view that represent today's phase of practical clinical knowledge for different cancer types. In the first two papers, we try to give a more organic and general evaluation of the issue. Then, for each specific cancer, no uniform criteria were requested to the authors to mirror the real current situation in continuous evolution. This collection of papers clearly shows the necessity to apply clinical research directly to today's patients because inter- and intra-tumour heterogeneity is so wide that any randomization process seems to be more or less unsatisfactory.

References

1 Nagel S, Borisch B, Thein SL, Oestreicher M, Nöthiger F, Birrer S, Tobler A, Fey MF: Somatic mutations detected by mini- and microsatellite DNA markers reveal clonal intratumor heterogeneity in gastrointestinal cancers. Cancer Res 1995;55:2866-2870.

2 Hasse U, Tinguely M, Leibundgut EO, Cajot JF, Garvin AM, Tobler A, Borisch B, Fey MF: Clonal loss of heterozygosity in microdissected Hodgkin and Reed-Sternberg cells. J Natl Cancer Inst 1999;91:1581-1583. 